\title{
FIRST-PRINCIPLES LCAO STUDY OF PHONONS IN NiWO
}

A. Kuzmin ${ }^{1 \dagger}$, A. Kalinko ${ }^{1}$, R.A. Evarestov ${ }^{2}$

${ }^{1}$ Institute of Solid State Physics, University of Latvia, Kengaraga street 8, LV-1063, Riga, Latvia

2 Department of Quantum Chemistry, St.Petersburg State University, 26 Universitetskiy Prospekt, Stary Peterhof 198504, Russia

\begin{abstract}
The electronic, structural and phonon properties of antiferromagnetic wolframite-type $\mathrm{NiWO}_{4}$ have been studied using first-principles spin-polarized LCAO calculations based on the hybrid Hartree-Fock (HF)/density functional (DFT) scheme. The influence of different percentages of HF contribution, i.e. different correlation strength, on the structure and phonon frequencies has been investigated and compared with the available experimental data.
\end{abstract}

PACS (2008): 71.15.Ap, 71.20.Ps, 75.50.Ee

Keywords: $\mathrm{NiWO}_{4} \cdot$ tungstates $\cdot \mathrm{LCAO} \cdot$ phonons $\bullet$ electronic structure

\footnotetext{
${ }^{\dagger}$ E-mail: a.kuzmin@cfi.lu.lv
} 


\section{Introduction}

Nickel tungstate $\left(\mathrm{NiWO}_{4}\right)$ has been extensively studied in the past because of its use for catalysts [1-4], humidity [5] and gas sensors [6], in microwave devices [7], and photoanodes [8, 9]. The application of mixed nickel-tungsten oxide thin films in electrochromic systems has also been proposed [10]. In such systems, the formation of the $\mathrm{NiWO}_{4}$ phase can occur at nano- or micro-scale resulting in a modification of their chromogenic properties. The properties of electrochromic materials are determined by charge insertion/extraction accompanied by the changes in the local atomic and electronic structures [11]. These phenomena have been studied for many years in pure $\mathrm{WO}_{3}$ and $\mathrm{NiO}$ both experimentally and theoretically [12-14], whereas the fundamental studies on mixed systems as well as pure $\mathrm{NiWO}_{4}$ are rather limited. In particular, to the best of our knowledge, the band structure calculations of $\mathrm{NiWO}_{4}$ have been not performed until now.

$\mathrm{NiWO}_{4}$ is isomorphous to other transition-metal tungstates such as $\mathrm{MnWO}_{4}, \mathrm{FeWO}_{4}$, $\mathrm{CoWO}_{4}$, and $\mathrm{ZnWO}_{4}$ [15], which, except for $\mathrm{ZnWO}_{4}$, have incomplete $3 \mathrm{~d}$ shell and are paramagnetic at room temperature. At low temperatures, $\mathrm{MnWO}_{4}, \mathrm{FeWO}_{4}$, $\mathrm{CoWO}_{4}$, and $\mathrm{NiWO}_{4}$ undergo cooperative transitions to antiferromagnetically-ordered states [16]. It should be noted that in the last few years $\mathrm{MnWO}_{4}$ has attracted increased attention as a multiferroic material [17].

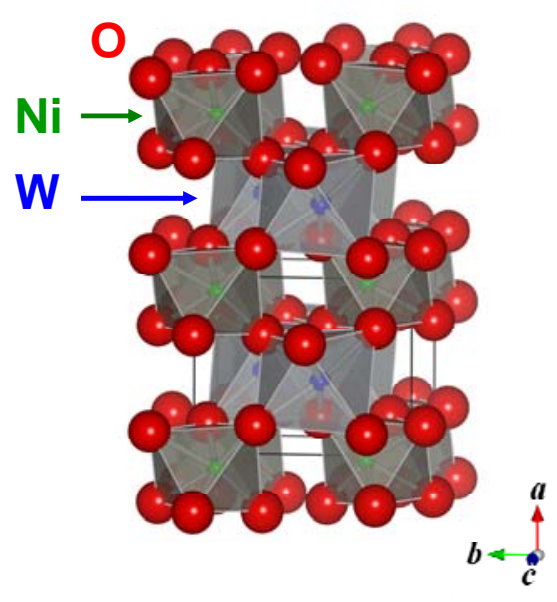

Figure 1. Crystal structure $(2 a, b, c)$ of monoclinic $(P 2 / c) \mathrm{NiWO}_{4}[15,18]$. The crystallographic unit cell is indicated. 
The crystal structure of $\mathrm{NiWO}_{4}$ has been determined at room temperature by x-ray [15] and neutron [18] powder diffraction. It is monoclinic wolframite-type with the space group $P 2 / c$. There are two formula units $(Z=2)$ per primitive cell, having the lattice parameters $a=4.5992 \AA, b=5.6606 \AA, c=4.9068 \AA$, and $\beta=90.03^{\circ}$ [18]. The presence of two non-equivalent oxygen atoms is responsible for three pairs of $\mathrm{Ni}-\mathrm{O}$ and $\mathrm{W}-\mathrm{O}$ bonds, having different lengths. Thus, both $\mathrm{Ni}$ and $\mathrm{W}$ atoms are surrounded by six oxygen atoms, forming distorted octahedral coordination, which has been directly probed by the $\mathrm{W} \mathrm{L}_{1,3}$ and $\mathrm{Ni} K$ edges $\mathrm{x}$-ray absorption spectroscopy in [19, 20]. Metal-oxygen octahedra of one type share edges and form zigzag chains along the c-axis (Fig. 1). The antiferromagnetic order exists in $\mathrm{NiWO}_{4}$ below $67 \mathrm{~K}$ [21]. Its magnetic unit cell $(2 a, b, c)$ is double the crystallographic one along the a-axis [16]. The spins at nickel ions in the same chain are arranged collinearly (coupled ferromagnetically), but are antiparallel (coupled antiferromagnetically) to the spins in adjacent chain.

In the present work, the electronic, structural and phonon properties of $\mathrm{NiWO}_{4}$ have been studied using first-principles spin-polarized periodic linear combination of atomic orbitals (LCAO) method with the hybrid Hartree-Fock (HF)-density functional (DFT) Hamiltonian. In this approach, basis functions for the crystalline orbitals are constructed as Bloch sums from the linear combination of localized Gaussian-type atomic orbitals. Therefore, a proper choice of the basis functions (preferably optimized for a particular system) is required. Such an optimized basis is used in the present calculations. The LCAO method allows one to use wavefunction-based (HF), density-based (DFT) and hybrid Hamiltonians (as PBE0 [22, 23] used in the present work). The latter provide the most accurate results eliminating the weaknesses of pure HF and DFT schemes, their realization within the Plane Wave basis calculations requires much bigger computational effort. LCAO methods are also able to avoid an artificial periodicity typically introduced in PW methods for a OD (cluster), 1D (wire), and 2D (slab) models.

\section{Computational details}

The methodology used in the present work has been employed by us recently to $\mathrm{ZnWO}_{4}$ in $[22,23]$, where the electronic, structural and phonon properties of tungstate have been studied within the periodic linear combination of atomic orbitals (LCAO) method [24]. The best agreement between experimental and theoretical 
structural and electronic parameters of $\mathrm{ZnWO}_{4}$ has been found for hybrid PBE0 (25\%) Hamiltonian [22, 23]. However, the infra-red (IR) and Raman phonon bands in $\mathrm{ZnWO}_{4}$ have been reproduced rather qualitatively [23]: in particular, the theory tends to overestimate the Raman phonon frequencies, especially, at high frequencies where the observed difference reaches $\sim 34 \mathrm{~cm}^{-1}$.

In this work we extend our studies to $\mathrm{NiWO}_{4}$, which represents an even more complicated case, since at low temperatures $(\leq 67 \mathrm{~K})$, it undergoes cooperative transition to an antiferromagnetically (AF)-ordered state [21]. The electronic, structural and phonon properties of $\mathrm{NiWO}_{4}$ have been studied using first-principles spin-polarized LCAO calculations by the CRYSTAL06 code [24]. To avoid core electrons of tungsten and nickel atoms, we used the Hay-Wadt (HW) effective small core potentials (ECP) and the corresponding atomic basis sets for metal atoms (W, $\mathrm{Ni}$ ), excluding diffuse Gaussian type orbitals. The basis set for tungsten was optimized by us for $\mathrm{ZnWO}_{4}$ [23], whereas the starting basis set for nickel was taken from [25] and re-optimized in the present work for $\mathrm{NiWO}_{4}$. The all-electron basis set, optimized in earlier calculations of perovskites [26], was used for oxygen atoms.

The calculations have been performed for four magnetic states by fixing the total spin projection. The first state (AF1), having the total spin projection $S_{z}=0$, corresponds to that observed experimentally $[18,21]$ with the ferromagnetic ordering of spins at nickel ions within zigzag chains but the antiferromagnetic alignment between different chains (Fig. 1). Note that AF1 state requires the doubling of crystallographic unit cell along a-axis direction $[18,21]$. There is also another possibility for antiferromagnetic state $(A F 2)$ with $S_{z}=0$ when spins at nickel ions in the same chain have antiparallel arrangement: in this case, no cell doubling is required since there are two nickel ions per primitive cell. The ferromagnetic (FM) state with all spins at nickel ions ordered collinearly and the non-magnetic (NM) state have also been calculated. In the CRYSTAL06 code, the accuracy in the evaluation of the Coulomb and exchange series is controlled by a set of tolerances, which were taken to be $\left(10^{-8}, 10^{-8}, 10^{-8}, 10^{-}\right.$ $\left.{ }^{8}, 10^{-16}\right)$ to provide high numerical accuracy. The Monkhorst-Pack scheme [27] for $8 \times 8 \times 8$ k-point mesh in the Brillouin zone was applied. The SCF calculations were performed for hybrid Hartree-Fock (HF)-DFT Hamiltonian (PBE0-type [28]) with different percentages $(0 \div 25 \%)$ of $\mathrm{HF}$ contribution. The calculations of phonon frequencies were done by the direct (frozen phonon) method [24]. The best agreement between calculated and experimental phonon frequencies has been 
observed for $13 \%$ HF contribution (PBE0 13\%), therefore only results for this case are given in Table 2 and will be discussed further.

\section{Results and discussion}

The equilibrium lattice parameters have been calculated by minimizing the crystal total energy for three magnetic (AF1, AF2 and FM) and non-magnetic (NM) states. Such simulations performed without accounting for any thermal effects are often referred as conducted at temperature $T=0 \mathrm{~K}$ [29]. The presence of thermal effects is expected to result in the lattice expansion and an increase of anharmonic contributions, thus shifting slightly the position of the vibrational bands and increasing their broadening. Both effects are known to be weak in tungstates [30, 31] and, in any case, are much smaller than accuracy of our calculations. In fact, weak sensitivity of the vibrational bands of tungstates to thermal effects finds practical application as Raman-active crystals in solid-state lasers, based on stimulated Raman scattering (SRS) [32, 33].

The calculated values of the structural parameters are compared in Table 1 with the experimental data obtained by neutron powder diffraction [18]. One can see that the results for magnetic states, having close total energies, agree better than $0.03 \AA$ with the experimental lattice constants and better than $0.1^{\circ}$ for the angle $\beta$.

The evaluated total energy per formula unit $E_{\text {tot }}$ (Table 1 ) has the lowest value for the antiferromagnetic state $\mathrm{AF} 1$ and the highest value for the non-magnetic state. The antiferromagnetic AF2 and ferromagnetic FM states have intermediate and close values of $E_{\text {tot, }}$ the latter being slightly lower. These results confirm the antiferromagnetic AF1-type ordering of spins in $\mathrm{NiWO}_{4}$ observed experimentally at low temperatures $[18,21]$. Note that in all calculated magnetic states the unpaired electrons are well localized at nickel atoms, giving a net atomic spin of about 1.70.

The Mulliken population analysis was used to estimate the total atomic charges. They are equal to $+1.67 \mathrm{e}$ for $\mathrm{Ni},+2.78 \mathrm{e}$ for $\mathrm{W},-1.16 \mathrm{e}$ for $\mathrm{O}_{1}$ and $-1.07 \mathrm{e}$ for $\mathrm{O}_{2}$ suggesting the mixed ionic-covalent character of the $\mathrm{Ni}-\mathrm{O}$ and $\mathrm{W}-\mathrm{O}$ bonding. In contrast to the purely ionic picture of a bare $\mathrm{W}^{6+}$ ion (with no valence electrons), the tungsten valence state in tungstate is around $3+$ due to back charge transfer from nearest oxygen atoms. This fact is responsible for a strong distortion of the $\mathrm{WO}_{6}$ octahedra caused by the second-order Jahn-Teller effect [34]. As a result, there are 
three significantly different $\mathrm{W}-\mathrm{O}$ bonds having lengths of about $1.81 \AA, 1.91 \AA$, and $2.12 \AA$ (Table 1).

Band structure diagram and spin dependent total/projected density of states for wolframite-type $\mathrm{NiWO}_{4}$ in the antiferromagnetic state are shown in Fig. 2. The dispersion curves are plotted along eight different symmetry directions in the Brillouin-zone. According to our calculations, $\mathrm{NiWO}_{4}$ has an indirect band gap $(\mathrm{E} \rightarrow \Gamma$ or $A \rightarrow \Gamma)$. The calculated band gap value $E_{g}=3.7 \mathrm{eV}$ is in good agreement with the experimental one equal to $3.6 \mathrm{eV}$ [8]. Note that the smaller value of the band gap in $\mathrm{NiWO}_{4}(2.28 \mathrm{eV}$ in [9] and $2.52 \mathrm{eV}$ in [35]) has been also reported. We believe that these two numbers are underestimated due to incorrect band gap determination in [9]. It seems that the low quality measurements performed in a limited energy range from $1.4 \mathrm{eV}$ to $3.4 \mathrm{eV}$ on $\mathrm{NiWO}_{4}$ thin films in [9] have led to confusion of the band gap with the absorption bands due to the $\mathrm{d}$-d transitions at $\mathrm{Ni}^{2+}$ ions, being responsible for the color of the compound $[8,36]$.

The calculated electronic structure in $\mathrm{NiWO}_{4}$ can be compared to that in other wolframite-type monoclinic $(P 2 / \mathrm{c})$ tungstates as $\mathrm{CdWO}_{4}$ [37], $\mathrm{FeWO}_{4}$ [38], $\mathrm{CoWO}_{4}$ [38], and $\mathrm{ZnWO}_{4}[22,23]$. as well as in triclinic $\mathrm{CuWO}_{4}$ [39]. Most previous firstprinciples calculations were based on DFT functionals and underestimate the band gap values: $E_{\mathrm{g}}=2.9 \mathrm{eV}$ versus $E_{\mathrm{g}}(\exp )=3.8-4.09 \mathrm{eV}$ in $\mathrm{CdWO}_{4}$ [37]; $E_{\mathrm{g}}=1.78 \mathrm{eV}$ versus $E_{g}(\exp )=2.0 \mathrm{eV}$ in $\mathrm{FeWO}_{4}$ [38]; $E_{\mathrm{g}}=1.36 \mathrm{eV}$ [38] versus $E_{g}(\exp )=2.0 \mathrm{eV}$ [40] in $\mathrm{CoWO}_{4} ; E_{g}=1.9 \mathrm{eV}$ [39] versus $E_{g}(\exp )=2.06 \mathrm{eV}$ [41] and $2.3 \mathrm{eV}[35,42]$ in $\mathrm{CuWO}_{4}$. However, the use of hybrid Hamiltonians within the LCAO method allowed us to obtain for $\mathrm{ZnWO}_{4}$ the band gap energy $E_{\mathrm{g}}=4.6 \mathrm{eV}$ [22] and $5.4 \mathrm{eV}$ [23] in better agreement with experimental values $E_{g}(\exp )=4.6 \mathrm{eV}$ [43] and $4.9 \mathrm{eV}$ [44].

Previous band structure calculations for these tungstates allows one to conclude that $\mathrm{FeWO}_{4}$ [38] and $\mathrm{CoWO}_{4}$ [38] have an indirect band-gap as we found also for $\mathrm{NiWO}_{4}$, whereas $\mathrm{CdWO}_{4}$ [37] and $\mathrm{ZnWO}_{4}[22,23]$ have a direct band-gap. Note that the indirect band-gap has been also found experimentally from optical absorption spectra in $\mathrm{CuWO}_{4}[35,42]$. The difference between the two groups of tungstates is connected with the contribution of partially filled $3 \mathrm{~d}$ states, provided by $\mathrm{Fe}^{2+}, \mathrm{Co}^{2+}, \mathrm{Ni}^{2+}$, and $\mathrm{Cu}^{2+}$ ions, into the band structure. In the case of $\mathrm{NiWO}_{4}$ (Fig. 2), the valence band is formed by hybridized $\mathrm{O} 2 \mathrm{p}$ and Ni3d states, and the $\mathrm{Ni3d}\left(\mathrm{t}_{2 \mathrm{~g}}, \mathrm{e}_{\mathrm{g}} \uparrow\right)$ states contribute mainly to the upper part of the valence band. The conduction band is formed by the empty W5d states with an admixture of the empty $\mathrm{Ni3d}\left(\mathrm{e}_{\mathrm{g}} \downarrow\right)$ states at the bottom of 
the band. These results agree well with those obtained recently by optical and ultraviolet photoelectron spectroscopy in [8].

Table 1. Lattice parameters $(a, b, c, \beta)$, atomic fractional coordinates $(x, y, z)$, total energy per formula unit $\left(E_{\text {tot }}\right)$ and the nearest interatomic distances ( $\mathrm{Ni}-\mathrm{O}$ and $\left.\mathrm{W}-\mathrm{O}\right)$, calculated for magnetic (AF1, AF2, FM) and non-magnetic (NM) states of wolframitetype $\mathrm{NiWO}_{4}$ using PBE0-type Hamiltonian (13\% HF). The room-temperature neutron powder diffraction (NPD) data [18] are shown for comparison.

\begin{tabular}{lccccc}
\hline & NPD & AF1 & AF2 & FM & NM \\
\hline $\mathrm{a}(\AA)$ & 4.5992 & 4.6226 & 4.6240 & 4.6247 & 4.5877 \\
$\mathrm{~b}(\AA)$ & 5.6606 & 5.6866 & 5.6874 & 5.6882 & 5.6084 \\
$\mathrm{c}(\AA)$ & 4.9068 & 4.9308 & 4.9319 & 4.9328 & 4.9518 \\
$\beta\left(^{\circ}\right)$ & 90.03 & 89.98 & 89.93 & 89.97 & 89.03 \\
\hline $\mathrm{Ni}(\mathrm{y})$ & 0.6616 & 0.6584 & 0.6583 & 0.6586 & 0.6541 \\
$\mathrm{~W}(\mathrm{y})$ & 0.1786 & 0.1798 & 0.1797 & 0.1800 & 0.1802 \\
$\mathrm{O} 1(\mathrm{x})$ & 0.2241 & 0.2189 & 0.2187 & 0.2187 & 0.2218 \\
$\mathrm{O} 1(\mathrm{y})$ & 0.1105 & 0.1071 & 0.1073 & 0.1071 & 0.1090 \\
$\mathrm{O} 1(\mathrm{z})$ & 0.9204 & 0.9320 & 0.9318 & 0.9322 & 0.9276 \\
$\mathrm{O} 2(\mathrm{x})$ & 0.2644 & 0.2590 & 0.2587 & 0.2586 & 0.2600 \\
$\mathrm{O} 2(\mathrm{y})$ & 0.3772 & 0.3776 & 0.3773 & 0.3779 & 0.3807 \\
$\mathrm{O} 2(\mathrm{z})$ & 0.3953 & 0.4038 & 0.4038 & 0.4035 & 0.4029 \\
\hline$E_{\text {tot }}(\mathrm{eV})$ & & -14625.740 & -14625.732 & -14625.734 & -14624.420 \\
\hline$R\left(\mathrm{Ni}-\mathrm{O}_{1}\right)(\AA)$ & 1.994 & 2.067 & 2.066 & 2.068 & 2.032 \\
$R\left(\mathrm{Ni}-\mathrm{O}_{2}\right)(\AA)$ & 2.061 & 2.049 & 2.051 & 2.053 & 2.027 \\
$R\left(\mathrm{Ni}-\mathrm{O}_{2}\right)(\AA)$ & 2.068 & 2.090 & 2.091 & 2.090 & 2.066 \\
\hline$R\left(\mathrm{~W}-\mathrm{O}_{2}\right)(\AA)$ & 1.803 & 1.809 & 1.809 & 1.809 & 1.814 \\
$R\left(\mathrm{~W}-\mathrm{O}_{1}\right)(\AA)$ & 1.957 & 1.911 & 1.911 & 1.911 & 1.921 \\
$R\left(\mathrm{~W}-\mathrm{O}_{1}\right)(\AA)$ & 2.107 & 2.119 & 2.120 & 2.121 & 2.114 \\
\hline & & & & &
\end{tabular}



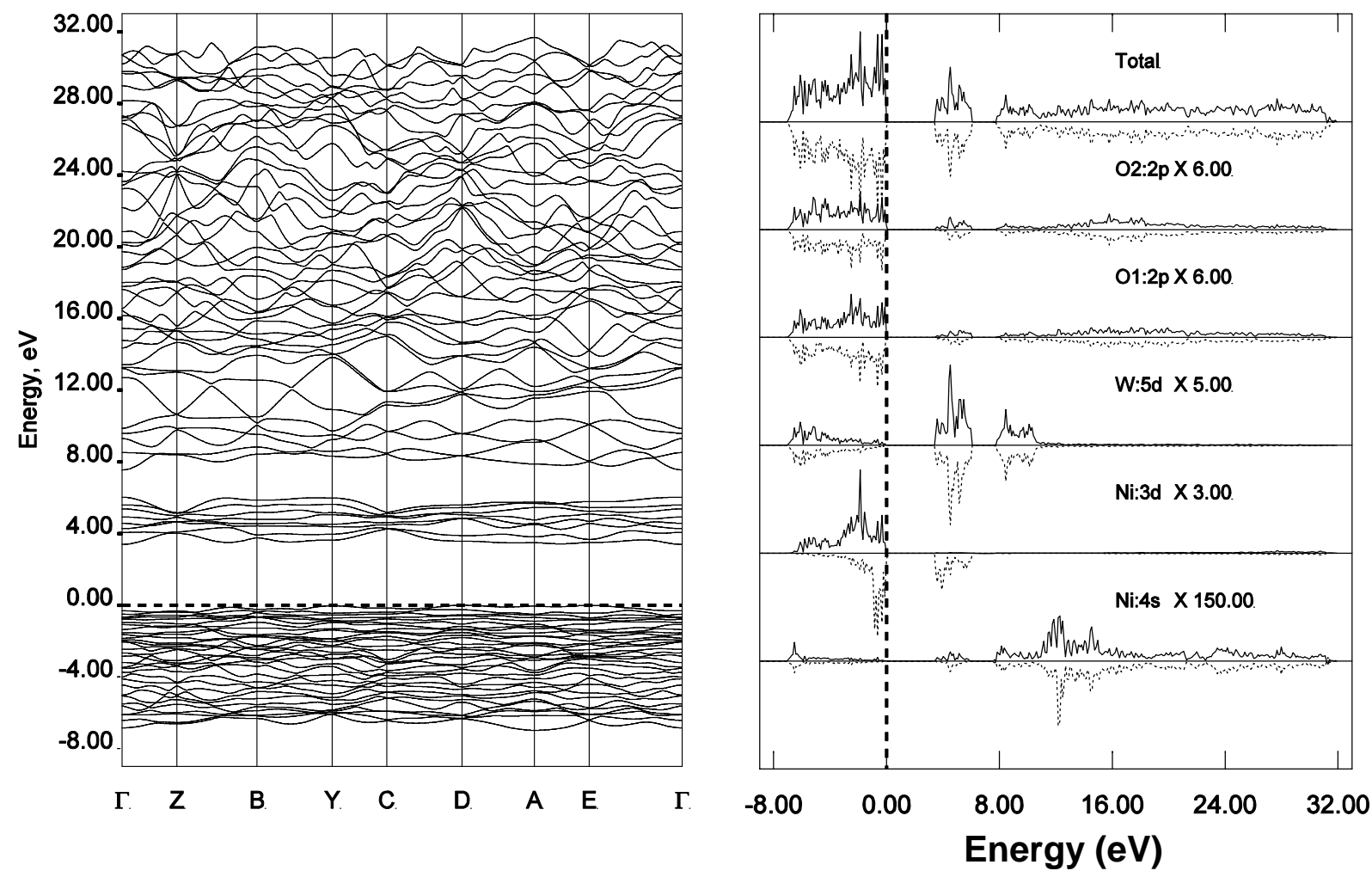

Figure 2. Band structure diagram (left panel) and spin dependent total/projected density of states (right panel) for wolframite-type $(P 2 / c) \mathrm{NiWO}_{4}$ in antiferromagnetic AF2 state. The energy zero is set at the Fermi energy level.
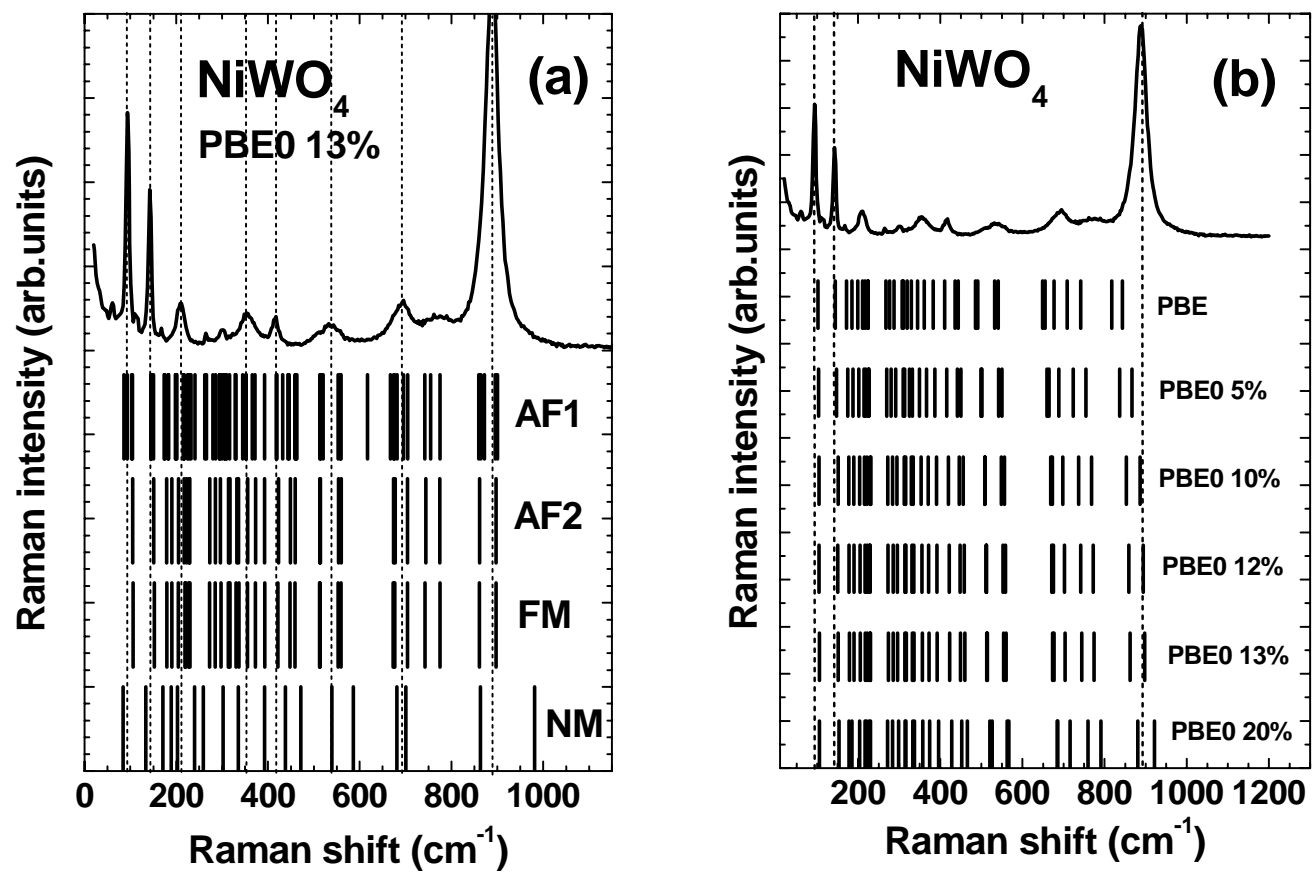

Figure 3. (a) Comparison of the room-temperature experimental Raman spectrum for $\mathrm{NiWO}_{4}$ with mode frequencies (vertical lines), calculated by the first-principles LCAO method using hybrid PBE0 13\% HF Hamiltonian for AF1, AF2, FM, and NM states. 
(b) LCAO results for hybrid PBE0-type Hamiltonian with different percentages $(0 \div 20 \%)$ of $\mathrm{HF}$ contribution in the AF2 state.

Table 2. Experimental and calculated (LCAO, PBE0-13\% exchange-correlation functional) zero pressure frequencies $(v)$ of the vibrational modes in $\mathrm{NiWO}_{4}$. Experimental frequencies ( $v_{\text {exper}}$ ) were obtained from the room-temperature Raman signal. The Raman and infra-red (IR) active(inactive) modes are denoted by $A(I)$ for non-magnetic (NM) and ferromagnetic (FM) phases. In antiferromagnetic AF2 phase all modes are both Raman and IR active. The first three acoustic modes (1, 2, and 3) are not given.

\begin{tabular}{|c|c|c|c|c|c|c|c|c|c|c|}
\hline \multirow[b]{2}{*}{$\begin{array}{l}\text { Phonon } \\
\text { mode }\end{array}$} & \multirow[b]{2}{*}{$\begin{array}{l}\begin{array}{l}\text { Vexper } \\
\left(\mathrm{cm}^{-1}\right)\end{array}\end{array}$} & \multirow[b]{2}{*}{$\begin{array}{c}\text { Phonon } \\
\text { symmetry }\end{array}$} & \multicolumn{3}{|c|}{ NM } & \multicolumn{3}{|c|}{ FM } & \multicolumn{2}{|c|}{ AF2 } \\
\hline & & & $\begin{array}{c}v^{v} \\
\left(\mathrm{~cm}^{-1}\right)\end{array}$ & Raman & IR & $\begin{array}{c}v \\
\left(\mathrm{~cm}^{-1}\right)\end{array}$ & Raman & IR & $\begin{array}{c}\text { Phonon } \\
\text { symmetry }\end{array}$ & $\begin{array}{c}v \\
\left(\mathrm{~cm}^{-1}\right)\end{array}$ \\
\hline 4 & 97 & $\mathrm{~B}_{\mathrm{a}}$ & 84 & $A$ & $\mathrm{I}$ & 84 & $A$ & $\mathrm{I}$ & $\mathrm{B}$ & 105 \\
\hline 5 & 149 & $A_{g}^{g}$ & 134 & A & I & 134 & A & I & A & 151 \\
\hline 6 & & $B_{u}$ & 143 & I & A & 143 & I & A & B & 179 \\
\hline 7 & 174 & $\mathrm{~B}_{\mathrm{q}}$ & 171 & A & I & 171 & A & I & B & 190 \\
\hline 8 & & $\mathrm{~B}_{\mathrm{u}}$ & 181 & 1 & A & 181 & I & A & B & 205 \\
\hline 9 & 197 & $\mathrm{~B}_{\mathrm{g}}$ & 189 & A & 1 & 189 & A & I & B & 217 \\
\hline 10 & 210 & $B_{g}$ & 202 & A & I & 202 & A & I & B & 222 \\
\hline 11 & 223 & $A_{g}^{g}$ & 240 & A & I & 240 & A & I & A & 228 \\
\hline 12 & 287 & $B_{g}^{g}$ & 259 & A & I & 259 & A & I & A & 229 \\
\hline 13 & & $B_{u}$ & 279 & 1 & A & 279 & I & A & B & 273 \\
\hline 14 & & $A_{u}$ & 283 & I & A & 283 & I & A & B & 285 \\
\hline 15 & 307 & $A_{g}$ & 302 & A & I & 302 & A & I & B & 296 \\
\hline 16 & & $\mathrm{~B}_{\mathrm{u}}$ & 317 & I & A & 317 & I & A & A & 314 \\
\hline 17 & 326 & $\mathrm{~B}_{\mathrm{q}}$ & 336 & A & I & 336 & A & I & B & 317 \\
\hline 18 & & $A_{u}$ & 358 & 1 & A & 358 & I & $A$ & B & 332 \\
\hline 19 & & $\mathrm{~B}_{\mathrm{u}}$ & 384 & I & A & 384 & I & A & A & 336 \\
\hline 20 & 363 & $A_{g}$ & 393 & A & 1 & 393 & A & I & A & 356 \\
\hline 21 & & $A_{u}^{g}$ & 402 & 1 & A & 402 & I & A & A & 373 \\
\hline 22 & & $A_{u}$ & 432 & i & A & 432 & i & A & B & 392 \\
\hline 23 & 380 & $\mathrm{~B}_{\mathrm{q}}$ & 438 & A & I & 438 & A & I & A & 423 \\
\hline 24 & 418 & $A_{q}$ & 471 & A & I & 471 & A & I & A & 449 \\
\hline 25 & & $\mathrm{~B}_{\mathrm{u}}$ & 494 & 1 & A & 494 & I & A & B & 460 \\
\hline 26 & & $\mathrm{~B}_{\mathrm{u}}$ & 523 & 1 & A & 523 & I & $A$ & $B$ & 513 \\
\hline 27 & & $A_{u}$ & 533 & 1 & A & 533 & I & A & A & 514 \\
\hline 28 & 512 & $\mathrm{~B}_{\mathrm{g}}$ & 539 & A & I & 539 & A & I & A & 554 \\
\hline 29 & 549 & $\mathrm{Ag}$ & 586 & A & i & 586 & A & I & $B$ & 560 \\
\hline 30 & 675 & $\mathrm{~B}_{\mathrm{g}}$ & 681 & A & I & 681 & A & I & B & 673 \\
\hline 31 & & $A_{u}$ & 681 & I & A & 681 & I & $A$ & $\bar{A}$ & 677 \\
\hline 32 & 697 & $A_{q}$ & 700 & A & 1 & 700 & A & I & A & 704 \\
\hline 33 & 775 & $\mathrm{~B}_{\mathrm{q}}$ & 863 & A & i & 863 & A & i & B & 744 \\
\hline 34 & & $\mathrm{~B}_{\mathrm{u}}$ & 877 & 1 & A & 877 & I & A & B & 775 \\
\hline 35 & & $A_{u}$ & 961 & I & A & 961 & I & A & A & 862 \\
\hline 36 & 893 & $A_{\mathrm{o}}$ & 982 & $A$ & I & 982 & $A$ & I & A & 897 \\
\hline
\end{tabular}


The calculated phonon frequencies (Table 2) are compared with the experimental Raman signal measured at room temperature in Fig. 3. According to group theoretical analysis, 36 phonon modes are expected at the Brillouin zone center $(\Gamma-$ point) for $\mathrm{NiWO}_{4}$ at room temperature. Among them 18 even modes $\left(8 \mathrm{~A}_{g}\right.$ and $\left.10 \mathrm{~B}_{g}\right)$ are Raman active. Note that the number of Raman active modes is doubled in the AF2 state and quadrupled in the AF1 state (Fig. 3a) due to the symmetry lowering caused by the spin ordering. Our results suggest that phonon frequencies are very sensitive to the type of the functional used in the calculations. In particular, for hybrid PBE0-type functional, composed of HF and DFT contributions, one can follow this dependence by varying the HF-to-DFT ratio (Fig. $3 \mathrm{~b}$ ). Our findings for $\mathrm{NiWO}_{4}$ suggest that $13 \% \mathrm{HF}$ admixture gives the best result for both lattice dynamics and the equilibrium structure.

One can compare the results for $\mathrm{NiWO}_{4}$ with first-principles phonon calculations performed for other tungstates. Most previous works were based on the plane-wave DFT approach, which results in underestimation of the high-frequency stretching WO mode by $\sim 45 \mathrm{~cm}^{-1}$ in $\mathrm{ZnWO}_{4}$ [45], $\sim 32 \mathrm{~cm}^{-1}$ in $\mathrm{CdWO}_{4}$ [46], and $\sim 52 \mathrm{~cm}^{-1}$ in $\mathrm{CuWO}_{4}$ [29] for PBE-type Hamiltonian [47] and by $\sim 4 \mathrm{~cm}^{-1}$ in $\mathrm{MgWO}_{4}$ [48] for simple LDA-type Hamiltonian [49]. On the contrary, our previous calculations [19] using hybrid PBE0 Hamiltonian [28] with 25\% HF admixture overestimated the frequencies of the stretching $\mathrm{W}-\mathrm{O}$ modes by $\sim 34 \mathrm{~cm}^{-1}$ in $\mathrm{ZnWO}_{4}$ and $\sim 39 \mathrm{~cm}^{-1}$ in $\mathrm{CaWO}_{4}$, which has the scheelite-type structure constructed of $\mathrm{WO}_{4}$ tetrahedra and $\mathrm{CaO}_{8}$ polyhedra. Our present calculations suggest that a variation of the HF admixture to the DFT Hamiltonian systematically influences the values of phonon frequencies (Fig. 3b). The best agreement with the high-frequency stretching $\mathrm{W}-\mathrm{O}$ mode in $\mathrm{NiWO}_{4}$ has been found for $13 \% \mathrm{HF}$ admixture, which results in the difference below $5 \mathrm{~cm}^{-1}$ (Table 2).

Finally we will discuss the distortion of the metal-oxygen octahedra (Table 1). The LCAO calculations predict larger distortion of the $\mathrm{WO}_{6}$ octahedra but weaker distortion of the $\mathrm{NiO}_{6}$ octahedra compared to diffraction [18]. In AF1 state, observed experimentally, the $\mathrm{NiO}_{6}$ octahedra are nearly regular according to the LCAO results 
and have three slightly different pairs of the $\mathrm{Ni}-\mathrm{O}$ bonds with the standard deviation of about $0.02 \AA$. On the contrary, the LCAO results for the $\mathrm{WO}_{6}$ octahedra predict three significantly different pairs of the $\mathrm{W}-\mathrm{O}$ bonds with the standard deviation of about $0.13 \AA$. The local distortion around metal atoms can be obtained experimentally by $x$-ray absorption spectroscopy (XAS), which is the local structural probe giving information complementary to diffraction methods [50]. In Fig. 4, we show our recent results obtained by the $\mathrm{WL}_{3}$-edge $X A S$ in $\mathrm{NiWO}_{4}$ at $10 \mathrm{~K}$ and $300 \mathrm{~K}$, measured at HASYLAB DESY synchrotron center in Hamburg. The use of regularization-like method [51,52] allowed us to reconstruct the radial distribution function (RDF) within the first coordination shell of tungsten (Fig. 4). The shape of the RDF unambiguously confirms the strong distortion of the $\mathrm{WO}_{6}$ octahedra and shows rather weak dependence on the temperature variation. Moreover, the comparison of the RDF with the lengths of the $\mathrm{W}-\mathrm{O}$ bonds, obtained by LCAO method in the present work and by neutron diffraction in [18], suggests that the LCAO calculations reproduce slightly better the position of the middle group of the $\mathrm{W}-\mathrm{O}$ distances at $1.911 \AA$. In fact, the value obtained by diffraction [18] $R(\mathrm{~W}-$ $\left.\mathrm{O}_{1}\right)=1.957 \AA$ is located in the valley region of the RDF. At the same time, the shortest and the longest $\mathrm{W}-\mathrm{O}$ bonds are very close in LCAO and diffraction.

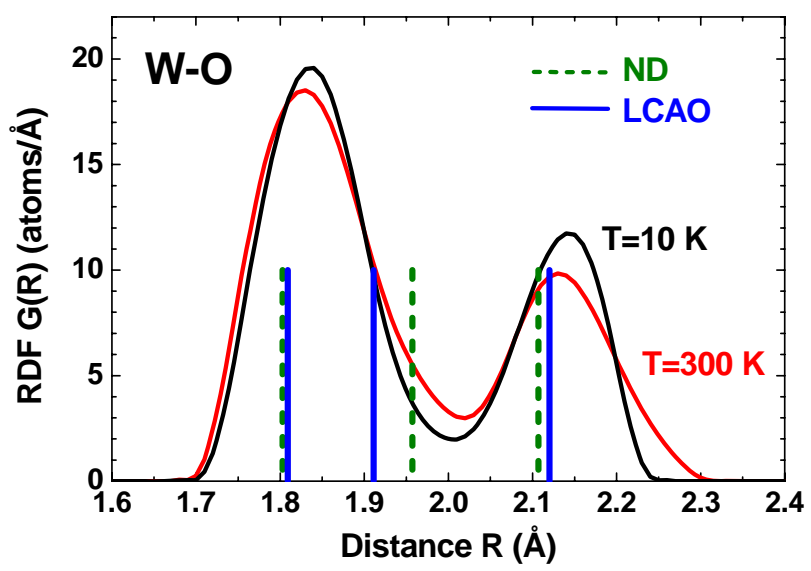

Figure 4. Comparison of the radial distribution functions (solid lines) for the first

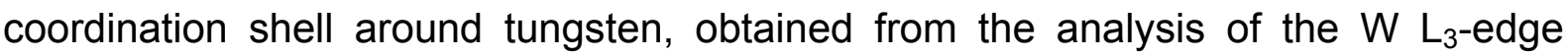
EXAFS spectra at $10 \mathrm{~K}$ and $300 \mathrm{~K}$, with the $\mathrm{W}-\mathrm{O}$ bond lengths obtained by neutron diffraction (ND) in [18] (dashed vertical lines) and LCAO calculation in the present work (solid vertical lines). 


\section{Conclusions}

The first-principles spin-polarized LCAO calculations have been performed for antiferromagnetic wolframite-type $\mathrm{NiWO}_{4}$. The indirect band gap of $E_{g}=3.7 \mathrm{eV}$ was calculated in good agreement with experiment. The variation of the HF/DFT mixing allowed us to determine the best HF admixture to be $\sim 13 \%$ : it results in a good reproduction of the crystal structure (including local octahedra distortions), band gap and phonon frequencies.

\section{Acknowledgements}

This work was supported by ESF Project 2009/0202/1DP/1.1.1.2.0/09/APIA/VIAA/141 and Latvian Government Research Grant No. 09.1518. The EXAFS measurements have been financed from the European Community's Seventh Framework Programme (FP7/2007-2013) under grant agreement No. 226716 (Project I-20090071 EC).

\section{References}

[1] F. J. Gil-Llambías, H. Rodriguez, I. Bouyssiers, M. Escudey, I. Carkovic, J. Catal. 102, 37 (1986)

[2] B. Scheffer, P. Molhoek, J. A. Moulijn, Appl. Catal. 46, 11 (1989)

[3] R. A. Diaz-Real, R. S. Mann, I. S. Sambi, Ind. Eng. Chem. Res. 32, 1354 (1993)

[4] D. L. Stern, R. K. Grasselli, J. Catal. 167, 570 (1997)

[5] A. K. Bhattacharya, R. G. Biswas, A. Hartridge, J. Mater. Sci. 32, 353 (1997)

[6] V. Dusastre, D. E. Williams, J. Mater. Chem. 9, 965 (1999)

[7] R. C. Pullar, S. Farrah, N. McN. Alford, J. Eur. Ceram. Soc. 27, 1059 (2007)

[8] T. Ejima, T. Banse, H. Takatsuka, Y. Kondo, M. Ishino, N. Kimura, M. Watanabe, I. Matsubara, J. Lumin. 119-120, 59 (2006)

[9] P. S. Pandey, N. S. Bhave, R. B. Kharat, Electrochim. Acta 51, 4659 (2006)

[10] A. Kuzmin, J. Purans, R. Kalendarev, D. Pailharey, Y. Mathey, Electrochim. Acta 46, 2233 (2001)

[11] G. A. Niklasson, C. G. Granqvist, J. Mater. Chem. 17, 127 (2007)

[12] S. K. Deb, Solar Energy Mater. Solar Cells 92, 245 (2008)

[13] K. Bange, Solar Energy Mater. Solar Cells 58, 1 (1999)

[14] S. Hüfner, Adv. Phys. 43, 183 (1994)

[15] R. O. Keeling, Jr., Acta Cryst. 10, 209 (1957) 
[16] C. P. Landee, E. F. Westrum, Jr., J. Chem. Thermodyn. 8, 471 (1976)

[17] O. Heyer, N. Hollmann, I. Klassen, S. Jodlauk, L. Bohaty, P. Becker, J. A. Mydosh, T. Lorenz, D. Khomskii, J. Phys.: Condens. Matter 18, L471 (2006)

[18] H. Weitzel, Z. Kristallogr. 144, 238 (1976)

[19] A. Kuzmin, J. Purans, R. Kalendarev, Ferroelectrics 258, 21 (2001)

[20] A. Kuzmin, J. Purans, Radiat. Meas. 33, 583 (2001)

[21] H. Weitzel, Solid State Commun. 8, 2071 (1970)

[22] A. Kalinko, A. Kuzmin, R. A. Evarestov, Solid State Commun. 149, 425 (2009)

[23] R. A. Evarestov, A. Kalinko, A. Kuzmin, M. Losev, J. Purans, Integr. Ferroelectr. 108, 1 (2009)

[24] R. Dovesi et al., Crystal 06, Users manual. (University of Turin, 2006)

[25] F. Freyria Fava, Ph. D'Arco, R. Orlando, R. Dovesi, J. Phys.: Condens. Matter 9, 489 (1997)

[26] S. Piskunov, E. Heifets, R. I. Eglitis, G. Borstel, Comput. Mater. Sci. 29, 165 (2004)

[27] H. J. Monkhorst, J. D. Pack, Phys. Rev. B 13, 5188 (1976)

[28] J. P. Perdew, M. Ernzerhof, K. Burke, J. Chem. Phys. 105, 9982 (1996)

[29] J. Ruiz-Fuertes, D. Errandonea, R. Lacomba-Perales, A. Segura, J. González, F.

Rodríguez, F. J. Manjón, S. Ray, P. Rodríguez-Hernández, A. Muñoz, Zh. Zhu, C. Y.

Tu, Phys. Rev. B 81, 224115 (2010)

[30] H. Wang, F. D. Medina, Y. D. Zhou, Q. N. Zhang, Phys. Rev. B 45, 10356 (1992) [31] M. N. lliev, M. M. Gospodinov, A. P. Litvinchuk, Phys. Rev. B 80, 212302 (2009) [32] A. A. Kaminskii, H. J. Eichler, K. Ueda, N. V. Klassen, B. S. Redkin, L. E. Li, J. Findeisen, D. Jaque, J. Garcia-Sole, J. Fernandez, R. Balda, Applied Optics 38, 4533 (1999)

[33] H. M. Pask, Prog. Quant. Electron. 27, 3 (2003)

[34] A. D. Walkingshaw, N. A. Spaldin, E. Artacho, Phys. Rev. B 70, 165110 (2004)

[35] R. Lacomba-Perales, J. Ruiz-Fuertes, D. Errandonea, D. Martínez-García, A. Segura, Europhys. Lett. 83, 37002 (2008)

[36] A. L. M. de Oliveira, J. M. Ferreira, M. R. S. Silva, G. S. Braga, L. E. B. Soledade, M. A. M. M. Aldeiza, C. A. Paskocimas, S. J. G. Lima, E. Longo, A. G. Souza, I. M. G. Santos, Dyes and Pigments 77, 210 (2008) [37] Y. Abraham, N. A. W. Holzwarth, R. T. Williams, Phys. Rev. B 62, 1733 (2000) 
[38] S. Rajagopal, V. L. Bekenev, D. Nataraj, D. Mangalaraj, O. Yu. Khyzhun, J. Alloys Compounds 496, 61 (2010)

[39] O. Yu. Khyzhun, V. L. Bekenev, Yu. M. Solonin, J. Alloys Compounds 480, 184 (2009)

[40] R. Bharati, R. A. Singh, B. M. Wanklyn, J. Mater. Sci. 16, 775 (1981)

[41] F. A. Benko, C. L. MacLaurin, F. P. Koffyberg, Mater. Res. Bull. 17, 133 (1982)

[42] J. Ruiz-Fuertes, D. Errandonea, A. Segura, F. J. Manjón, Zh. Zhu, C. Y. Tu, High Press. Research 28, 565 (2008)

[43] V. N. Kolobanov, I. A. Kamenskikh, V. V. Mikhailin, I. N. Shpinkov, D. A. Spassky, B. I. Zadneprovsky, L. I. Potkin, G. Zimmerer, Nucl. Instrum. Methods Phys. Res. A 486, 496 (2002)

[44] M. Itoh, N. Fujita, Y. Inabe, J. Phys. Soc. Jap. 75, 084705 (2006)

[45] D. Errandonea, F. J. Manjón, N. Garro, P. Rodríguez-Hernández, S. Radescu, A. Mujica, A. Muñoz, C. Y. Tu, Phys. Rev. B 78, 054116 (2008)

[46] R. Lacomba-Perales, D. Errandonea, D. Martinez-Garcia, P. RodríguezHernández, S. Radescu, A. Mujica, A. Muñoz, J. C. Chervin, A. Polian, Phys. Rev. B 79, 094105 (2009)

[47] J. P. Perdew, K. Burke, M. Ernzerhof, Phys. Rev. Lett. 77, 3865 (1996)

[48] J. Ruiz-Fuertes, S. López-Moreno, D. Errandonea, J. Pellicer-Porres, R. Lacomba-Perales, A. Segura, P. Rodríguez-Hernández, A. Muñoz, A. H. Romero, J. González, J. Appl. Phys. 107, 083506 (2010)

[49] J. P. Perdew, A. Zunger, Phys. Rev. B 23, 5048 (1981)

[50] J. J. Rehr, R. C. Albers, Rev. Mod. Phys. 72, 621 (2000)

[51] A. Kuzmin, Physica B 208/209, 175 (1995)

[52] A. Kuzmin, J. Purans, J. Phys.: Condens. Matter 12, 1959 (2000) 